\title{
A REMARK ON THE DISTRIBUTIVE LAW FOR AN IDEAL IN A COMMUTATIVE RING
}

\author{
by C. U. JENSEN
}

(Received 13 August 1965)

Let $R$ be a commutative ring. with an identity element. It is the purpose of this note to establish conditions for an arbitrary but fixed ideal a of $R$ to satisfy the distributive law

$$
\mathfrak{a} \cap(\mathfrak{b}+\mathfrak{c})=a \cap b+a \cap c
$$

for all ideals $\mathfrak{b}$ and $\mathfrak{c}$ of $R$. In particular, in the Noetherian case, this will be related to the decomposition of $a$ into prime ideals. We start with

Proposition 1. For a fixed ideal $\mathfrak{a}$ in a commutative ring $R$ with an identity element, the following conditions are equivalent.

(i) $\mathfrak{a} \cap(\mathfrak{b}+\mathrm{c})=\mathfrak{a} \cap \mathfrak{b}+\mathfrak{a} \cap c$ for any two ideals $\mathfrak{b}$ and $\mathrm{c}$ of $R$.

(ii) $\mathfrak{a}+\mathfrak{b} \cap \mathfrak{c}=(\mathfrak{a}+\mathfrak{b}) \cap(\mathfrak{a}+\mathfrak{c})$ for any two ideals $\mathfrak{b}$ and $\mathfrak{c}$ of $R$.

(iii) For any maximal ideal $m$ of $R$, the ideals of the local (generalized) quotient ring $R_{\mathfrak{t r}}$ are separated by a $R_{2 m}$, i.e., any ideal of $R_{\mathfrak{2 1}}$ is either contained in $\mathfrak{a} R_{\mathfrak{m}}$ or contains $\mathfrak{a} R_{\mathfrak{m}}$.

Proof. Since an ideal a of $R$ is uniquely determined by its local components $a R_{217}$ and the formation of sums and intersections is preserved by extension from $R$ to $R_{\mathfrak{m}}$, it is readily seen that (iii) implies (i) and (ii).

By extension of the ideals from $R$ to $R_{2 m}$, the implication (i) $\Rightarrow$ (iii) is proved by showing that an ideal $a$ of a local ring $R$ for which (i) holds, will separate the ideals of $R$. To this end, it suffices to prove that, for any element $b \in R, b \notin a$, the principal ideal $b R$ generated by $b$ will contain $\mathfrak{a}$. In fact, let $a$ be an arbitrary element in $\mathfrak{a}$ and put $\mathfrak{b}=b R, \mathfrak{c}=(a-b) R$. Then $a \in \mathrm{a} \cap(\mathrm{b}+\mathrm{c})$ and (i) implies the representation of $a$ as

$$
a=b x+(a-b) y \text {, where } x, y \in R, \quad b x \in \mathfrak{a}, \quad(a-b) y \in \mathfrak{a} .
$$

Now, $b y \in \mathfrak{a}, b \notin \mathfrak{a}$; so $y$ is a non-unit in $R$. Since $R$ is local, $1-y$ is a unit in $R$, and

$$
a(1-y)=b(x-y)
$$

thus implies that $a \in b R$. This holds for any element $a \in \mathfrak{a}$; whence $a \subseteq b R$.

The implication (ii) $\Rightarrow$ (iii) may be proved similarly. For convenience we introduce the following

Definition. An ideal a in a commutative ring $R$ is called a $D$-ideal if it satisfies one and hence all of the conditions in Proposition 1.

For finitely generated ideals in an integral domain we can give some further characterizations of $D$-ideals. 
Proposition 2. Let a be an arbitrary finitely generated ideal of an integral domain $R$. Then a is a $D$-ideal if and only if one of the following equivalent conditions is fulfilled.

(iv) $(\mathfrak{a}: \mathfrak{b})+(\mathfrak{b}: \mathfrak{a})=R$ for all finitely generated ideals $\mathfrak{b}$ of $R$.

(v) $(\mathfrak{a}+\mathfrak{b})(\mathfrak{a} \cap \mathfrak{b})=\mathfrak{a} \mathfrak{b}$ for all ideals $\mathfrak{b}$ of $R$.

REMARK. In particular, (iv) and (v) give characterizations of $D$-ideals in Noetherian domains.

Proof. Since $(\mathrm{a}: \mathrm{b}) R_{\mathfrak{m}}=\left(\mathfrak{a} R_{\mathfrak{m}}: \mathfrak{b} R_{\mathrm{m}}\right)$ for finitely-generated ideals, it is easily seen by condition (iii) in Proposition 1 that any finitely-generated $D$-ideal satisfies (iv) and (v).

Conversely, let $a$ be a finitely generated ideal satisfying (iv). By extension of the ideals from $R$ to $R_{\mathfrak{m}}$ (m maximal), we may without restriction assume that $R$ is local. We then have to prove that $a \subseteq b R$ for any $b \in R, b \notin \mathfrak{a}$. Because of the relation

$$
(a: b R)+(b R: \mathfrak{a})=R,
$$

we have a decomposition of the identity element 1 :

$$
1=x+y, \quad x \in(\mathfrak{a}: b R), \quad y \in(b R: \mathfrak{a}) .
$$

Since $b \notin \mathfrak{a}, x$ must be a non-unit and hence $y$ a unit, which implies that $a \subseteq b R$. Thus $a$ separates the ideals of $R$, and $a$ is a $D$-ideal.

Finally let a satisfy (v). As before we may assume that $R$ is local. Let $b$ be an arbitrary element in $R, b \notin a$. Because of $(\mathrm{v})$, we have

$$
(a+b R)(a \cap b R)=b a \text {. }
$$

For any element $a \in a$ we get a representation of $a b$ in the form

$$
a b=b x+\sum_{i} a_{i} y_{i} \quad\left(a_{i} \in \mathfrak{a}, \quad y_{i} \in \mathfrak{a} \cap b R, \quad x \in \mathfrak{a} \cap b R\right) .
$$

As elements of $b R, x$ and $y_{i}$ may be written as

$$
x=b r, \quad y_{i}=b r_{i} \quad\left(r \in R, \quad r_{i} \in R\right) .
$$

Since $y_{i} \in a$ and $b \notin a$, the elements $r_{i}$ must be non-units and thus belong to the unique maximal ideal of $R$, say $m$. From (1) and (2) we derive the relation

$$
a=b r+\sum_{i} a_{i} r_{i}
$$

This means that $a \in b R+a \mathfrak{m}$, because $r_{i} \in \mathfrak{m}$. This holds for any element $a \in \mathfrak{a}$, and thus $a \subseteq b R+a m$. Since $a$ is finitely generated, we conclude by a well-known argument (Nakayama's lemma, [3, Theorem 4.1]) that $a+b R=b R$ or $\mathfrak{a} \subseteq b R$. Consequently a separates the ideals of $R$ and $a$ is a $D$-ideal. The proof of Proposition 2 is now complete.

Before stating the next theorem we shall prove

LEMma 1. In a Noetherian local ring $R$ with maximal ideal $\mathrm{m}$, any non-zero $D$-ideal is a power $m^{v}$ of $m$. 
Proof. Let $a \neq(0)$ be a $D$-ideal in $R$. In the Noetherian ring $R$ we have

$$
\bigcap_{v=1}^{\infty} \mathfrak{m}^{v}=(0) \text {. }
$$

Consequently there exists a $v$ such that $\mathfrak{a} \subseteq \mathfrak{m}^{\nu}, \mathfrak{a} \$ \mathfrak{m}^{\nu+1}$. Since $\mathfrak{a}$ is a $D$-ideal in the local ring $R$, we see that $a \nsubseteq \mathfrak{m}^{v+1}$ implies that $\mathfrak{m}^{v+1} \subseteq a$. Suppose that $a \neq \mathfrak{m}^{v}$ and $a \neq \mathfrak{m}^{v+1}$. There will then be elements $a \in \mathfrak{a}, a \notin \mathfrak{m}^{v+1}$ and $x \in \mathfrak{m}^{v}, x \notin a$. Since a separates the ideals of $R$, it follows that $x \notin \mathfrak{a}$ implies that $\mathfrak{a} \subseteq x R$; hence $a=x r$. Here $r$ must be a non-unit, i.e. $r \in \mathfrak{m}$, because $x \notin a$. Hence $a \in m^{v} \mathfrak{m}=\mathrm{m}^{v+1}$, contradicting the assumption about $a$. Thus $a$ is a power of $\mathrm{m}$.

THEOREM 1. Any non-zero $D$-ideal in a Noetherian integral domain $R$ may be written uniquely as a product of ( finitely many) maximal ideals.

Proof. For any maximal ideal $\mathfrak{m}$ containing the $D$-ideal $\mathfrak{a}$, the preceding lemma shows that $a R_{\mathrm{m}}$ has the form $\mathrm{m}^{v} R_{\mathrm{m}}$ for a suitable $v$. As an ideal of a Noetherian ring, $a$ has a primary decomposition in which the prime ideals belonging to the various primary components are all different:

$$
\mathfrak{a}=\bigcap_{i=1}^{n} \mathfrak{q}_{i} .
$$

Suppose that $\mathrm{q}_{t} \subseteq \mathrm{m}$ for $1 \leqq i \leqq k(\leqq n)$ and that $\mathrm{q}_{i} \ddagger \mathrm{m}$ for $k<i$; then

$$
\mathfrak{m}^{v} R_{\mathfrak{m}}=\mathfrak{a} R_{\mathfrak{m}}=\bigcap_{i=1}^{k} q_{i} R_{\mathfrak{m}}
$$

Identifying the radicals of the two sides, we get

$$
m R_{\mathfrak{m}}=\bigcap_{i=1}^{k}\left(\operatorname{Rad} \mathfrak{q}_{i}\right) R_{\mathfrak{m l}},
$$

and hence $k=1, \operatorname{Rad} q_{1}=m$. This implies that $q_{1}=m^{\nu}$. Consequently, since any $q_{i}$ is contained in a maximal ideal, $\mathfrak{a}$ is represented as an intersection of powers of different maximal ideals. Since these are pairwise comaximal, the intersection is equal to the product of the powers of the maximal ideals. The uniqueness follows in a well known way by extension to the quotient rings $R_{\mathfrak{m}}(\mathfrak{a} \subseteq \mathfrak{m})$.

Remark. Theorem 1 implies in particular that a Noetherian domain in which any ideal is a $D$-ideal (i.e., in which the lattice of all ideals is distributive) is a Dedekind domain (cf. [1, Theorem 8]). If $R$, moreover, is assumed to be integrally closed in its quotient field, the $D$-ideals can be determined more precisely.

Proposition 3. Let $R$ be an integrally closed Noetherian local domain with maximal ideal $m$. If there exists a non-trivial $D$-ideal a (i.e. $a \neq(0), a \neq m, a \neq R)$, then $R$ is regular of dimension 1, i.e., $R$ is a principal ideal domain in which any proper ideal is a power of $\mathfrak{m}$. 
Proof. It is sufficient to show that $m$ is principal, since this implies that $\operatorname{dim} R / \mathfrak{m}\left(\mathfrak{m} / \mathfrak{m}^{2}\right)=1$. Let $x_{1}, \cdots, x_{s}$ be a minimal base of $m$. According to Lemma $1, \mathfrak{a}$ has the form $\mathfrak{a}=\mathfrak{m}^{r}$, where $r \geqq 2$. Now, as an element of a minimal base of $m, x_{1}$ cannot belong to $\mathfrak{a}$; consequently, since $\mathfrak{a}$ is a $D$-ideal, we have $\mathfrak{a}=\mathfrak{m}^{r} \subseteq x_{1} R$.

For an arbitrary element $y_{r-1} \in \mathfrak{m}^{r-1}$, we have $y_{r-1} \mathfrak{m} \subseteq \mathfrak{m}^{r} \subseteq x_{1} R$; hence $\left(y_{r-1} / x_{1}\right) \mathrm{m}$ is either $R$ or an ideal contained in $\mathfrak{m}$. If $\left(y_{r-1} / x_{1}\right) \mathfrak{m}=R$, it is obvious that $m$ is principal and the proof is complete. If $\left(y_{r-1} / x_{1}\right) \mathrm{m} \subseteq \mathrm{m}$, we conclude that $y_{r-1} / x_{1} \in R$, since $m$ is finitely generated and $R$ is integrally closed in its quotient field. Thus we may assume that $\mathrm{m}^{r-1} \subseteq x_{1} R$; by repeating the above argument we see that $m$ is either principal or $m^{r-2} \subseteq x_{1} R$. Continuing in this way, we find that $m$ is principal or $m \subseteq x_{1} R$; but, since $x_{1}$ is an element in $m$, this also implies that $m$ is principal.

Before formulating the next theorem, we recall [2] that the fundamental theorem of ideal theory is said to hold for an ideal $\mathfrak{a}$ of $R$ if $\mathfrak{a}$ is a product of prime ideals

$$
a=p_{1}^{k_{1}} \cdots p_{r}^{k_{r}} \quad\left(p_{i} \neq p_{j} \text { for } i \neq j\right)
$$

and if, further, $\mathrm{a} \subseteq \mathrm{b} \subset R$ implies that $\mathrm{b}=\mathfrak{p}_{1}^{l_{1}} \cdots \mathfrak{p}_{k}^{l_{k}}$ with $0 \leqq l_{i} \leqq k_{i}$.

THEOREM 2. Let $R$ be an integrally closed Noetherian domain. For an arbitrary but fixed non-zero ideal a of $R$, the following conditions are equivalent.

(i) a is a D-ideal.

(ii) $\mathfrak{a}$ and any proper ideal $\mathfrak{b} \supseteq \mathfrak{a}$ are products of maximal ideals.

(iii) The fundamental theorem of ideal theory holds for a.

Proof. (i) $\Rightarrow$ (ii). a is a $D$-ideal if and only if $a R_{\mathfrak{m}}$ is a $D$-ideal in $R_{\mathfrak{m}}$ for any maximal ideal $\mathrm{m}$. Proposition 3 implies that all overideals of a non-zero $D$-ideal in an integrally closed Noetherian local domain are $D$-ideals, and hence that any ideal $\mathfrak{b}$ in $R$, with $\mathfrak{b} \supseteq \mathfrak{a}$, is a $D$-ideal. (ii) is then a consequence of Theorem 1.

(ii) $\Rightarrow$ (iii). Suppose that $\mathfrak{a}$ is a product of maximal ideals:

$$
\mathfrak{a}=\mathfrak{m}_{1}^{v_{1}} \ldots \mathfrak{m}_{r}^{v_{r}} \quad\left(\mathfrak{m}_{i} \neq \mathfrak{m}_{j} \text { for } i \neq j\right) .
$$

Any ideal $\mathfrak{b} \supseteq \mathfrak{a}$ is a product of maximal ideals. Considering the extensions to $R_{\mathfrak{m}}(\mathrm{m}$ maximal), we see that the representation of $\mathfrak{b}$ as a product of maximal ideals must have the form

$$
\mathfrak{b}=\mathrm{m}_{1}^{\mu_{1}} \cdots \mathrm{m}_{r}^{\mu_{r}} \quad\left(0 \leqq \mu_{i} \leqq v_{i}\right) .
$$

Hence the fundamental theorem of ideal theory holds for $a$.

(iii) $\Rightarrow$ (i). It is obvious for an ideal $a$ satisfying (iii) that there are only finitely many ideals $\mathfrak{b} \supseteq \mathfrak{a}$. In particular, for any prime ideal $\mathfrak{p} \supseteq \mathfrak{a}$, we have that $R / \mathfrak{p}$ contains only finitely many ideals, i.e., $p$ is maximal. Therefore $a$ is a product of maximal ideals,

$$
a=m_{1}^{k_{1}} \cdots m_{r}^{k_{r}} \quad\left(m_{i} \neq m_{j} \text { for } i \neq j\right),
$$

and any ideal $\mathfrak{b} \supseteq a$ has the form $\mathfrak{b}=m_{1}^{l_{1}} \cdots m_{r}^{l_{r}}\left(0 \leqq l_{i} \leqq k_{i}\right)$. 
Since $a R_{\mathfrak{m}}=R_{\mathfrak{m}}$ for any $\mathrm{m} \neq \mathrm{m}_{i}$, it suffices to show that $\mathfrak{a} R_{\mathrm{mm}_{i}}$ separates the ideals of $R_{\mathrm{m}_{\mathrm{i}}}$ for $i=1, \ldots, r$. If $k_{i}=1$, then $\mathrm{a} R_{\mathrm{m}_{i}}=\mathrm{m}_{i} R_{\mathrm{m}_{i}}$ clearly is a $D$-ideal of $R_{\mathfrak{m}_{i}}$ If $k_{i}>1$, then the powers $\mathfrak{m}_{i}^{l_{t}}\left(0 \leqq l_{i} \leqq k_{i}\right)$ are the only ideals containing $\mathrm{m}_{i}^{k_{i}}$. This implies that there is no ideal lying properly between $\mathrm{m}_{i} R_{\mathrm{m}_{1}}$ and $\mathrm{m}_{i}^{2} R_{\mathrm{m}_{i}}$. Hence $\operatorname{dim}_{R_{\mathrm{m}_{1}} / \mathrm{m}_{1} R_{\mathrm{m}_{i}}}\left(\mathrm{~m}_{i} R_{\mathrm{m}_{i}} / \mathrm{m}_{i}^{2} R_{\mathrm{m}_{i}}\right)=1$ and $R_{\mathrm{m}_{1}}$ is a regular local ring of dimension 1. In this case all proper ideals of $R_{\mathrm{m}_{1}}$ are powers of $\mathrm{m}_{i} R_{\mathrm{m}_{i}}$; in particular $\mathrm{a} R_{\mathrm{m}_{i}}=\mathrm{m}_{i}^{k_{i}} R_{\mathrm{m}_{i}}$ separates the ideals of $R_{\mathrm{m}_{i}}$.

We shall finish by considering commutative Noetherian rings, the prime ideals of which are $D$-ideals. For this purpose we need the following lemma.

LEMmA 2. A prime ideal $\mathfrak{p}$ in a commutative Noetherian ring is a D-ideal if and only if $\mathfrak{p} R_{\mathrm{ll}}=(0)$ or $\mathfrak{m} R_{\mathfrak{m}}$ for any maximal ideal $\mathfrak{m}$ of $R$.

Proof. The "if " part is clear. To prove the converse it is enough to show that a prime $D$-ideal $\mathfrak{p}$ in a Noetherian local ring $R$ is equal to (0) or the maximal ideal $m$ of $R$. This, however, is an immediate consequence of Lemma 1, which implies that $p$ has the form $\mathfrak{p}=\mathrm{m}^{v}$ for a suitable $v$. But, when $\mathfrak{p}$ is prime, $v$ must be 1 .

We are now able to prove

THEOREM 3. Let $R$ be a commutative Noetherian ring with an identity element. The prime ideals of $R$ are all of them $D$-ideals if and only if $R$ is a direct sum of integral domains of Krulldimension $\leqq 1$ and of primary rings (local rings of dimension zero). Equivalently, the prime ideals of $R$ are $D$-ideals if and only if $R$ is a direct sum of rings with restricted minimum condition (i.e. rings for which any proper residue class ring satisfies the descending chain condition).

Proof. Any prime ideal in a ring with restricted minimum condition is either (0) or maximal, hence a $D$-ideal. Moreover, it is readily checked that the direct sum of rings for which any prime ideal is a $D$-ideal has this property itself. This makes the "if " part in both formulations obvious. It is sufficient to prove the "only if " part in the first formulation, since integral domains of Krull-dimension $\leqq 1$ and primary rings always satisfy the restricted minimum condition (cf. [1]).

Now, let $R$ be a commutative Noetherian ring, the prime ideals of which are $D$-ideals. Since $R$ is Noetherian, the ideal $(0)$ has an irredundant primary representation $(0)=\bigcap_{i} q_{i}$. Let $\mathfrak{p}_{i}=\operatorname{Rad} q_{i}$. The prime ideals $\mathfrak{p}_{i}$ must be pairwise comaximal, for if $\mathfrak{p}_{i}$ and $\mathfrak{p}_{j}(i \neq j)$ were not comaximal, $\mathfrak{p}_{i}$ and $\mathfrak{p}_{j}$ would be contained in a maximal ideal $\mathrm{m}$. In $R_{\mathfrak{m}}$ we would have $\mathrm{p}_{i} R_{\mathrm{m}} \subseteq \mathrm{m} R_{\mathrm{m}}$ and $\mathrm{p}_{j} R_{\mathrm{m}} \subseteq \mathrm{m} R_{\mathrm{m}}$. The prime ideals in $R_{\mathrm{m}}$ are in 1-1 correspondence with the prime ideals of $R$ contained in $m$. This, together with Lemma 2, implies that, for example, $\mathfrak{p}_{i} R_{\mathrm{m}}=(0), \mathfrak{p}_{j} R_{\mathfrak{m}}=\mathfrak{m} R_{\mathfrak{m}}$, and hence that $\mathfrak{p}_{i} \subset \mathfrak{p}_{j}$. Since (0) is the only $\mathfrak{p}_{i} R_{\mathfrak{m}}$-primary ideal in $R_{\mathfrak{m}}$, it follows that $\mathrm{p}_{i}$ is the only $\mathrm{p}_{i}$-primary ideal of $R$; i.e., that $\mathfrak{q}_{i}=\mathfrak{p}_{i}$. Similarly, since any $\mathrm{m} R_{\mathrm{m}}$-primary ideal contains $(0)=\mathfrak{p}_{i} R_{\mathfrak{m}}$, any $\mathrm{m}$-primary ideal of $R$ contains $\mathfrak{p}_{i} ;$ i.e., $\mathfrak{q}_{j} \supset \mathfrak{p}_{i}=\mathfrak{q}_{l}$. This contradicts the irredundance.

Thus the ideals $p_{i}$ are pairwise comaximal, and this implies that the ideals $q_{i}$ are also pairwise comaximal. Hence [4, Chapter III, Theorem 32] $R$ is a direct sum of rings isomorphic to the rings $R / \mathfrak{q}_{i}$. If $\operatorname{Rad} \mathfrak{q}_{i}=\mathfrak{p}_{i}$ is maximal, then $R / \mathfrak{q}_{i}$ is a primary ring; if $\operatorname{Rad} q_{i}=\mathfrak{p}_{i}$ is not 
maximal, then the above argument shows that $q_{i}=p_{i}$ and any prime ideal properly containing $\mathfrak{p}_{i}$ is maximal. Hence $R / \mathfrak{q}_{i}=R / \mathfrak{p}_{i}$ is an integral domain of Krull-dimension $\leqq 1$.

\section{REFERENCES}

1. I. S. Cohen, Commutative rings with restricted minimum condition, Duke Math. J. 17 (1950), $27-42$.

2. L. Fuchs, On the fundamental theorem of commutative ideal theory, Acta Math. Acad. Sci. Hungar. 5 (1954), 95-99.

3. M. Nagata, Local rings (New York, 1962).

4. O. Zariski and P. Samuel, Commutative algebra, Vol. I (Princeton, 1958).

UNIVERSITY OF COPENHAGEN 